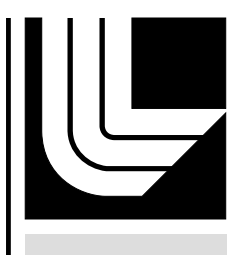

LAW RENCE LIVERMORE N A T IO N A L LABORATORY

\title{
UCRL-JRNL-235801
}

\section{An Alternative Model for Electron Correlation in $\mathrm{Pu}$}

S.W. Yu, J.G. Tobin, P. Soderlind

October 24, 2007

J. Phys. Cond. Matter- Fast Track Commun 
This document was prepared as an account of work sponsored by an agency of the United States government. Neither the United States government nor Lawrence Livermore National Security, LLC, nor any of their employees makes any warranty, expressed or implied, or assumes any legal liability or responsibility for the accuracy, completeness, or usefulness of any information, apparatus, product, or process disclosed, or represents that its use would not infringe privately owned rights. Reference herein to any specific commercial product, process, or service by trade name, trademark, manufacturer, or otherwise does not necessarily constitute or imply its endorsement, recommendation, or favoring by the United States government or Lawrence Livermore National Security, LLC. The views and opinions of authors expressed herein do not necessarily state or reflect those of the United States government or Lawrence Livermore National Security, LLC, and shall not be used for advertising or product endorsement purposes. 


\title{
An Alternative Model for Electron Correlation in $\mathrm{Pu}$
}

\author{
S.W. Yu, J.G. Tobin*, and P. Söderlind \\ Lawrence Livermore National Laboratory, LLNS-LLC, Livermore, CA, USA
}

Abstract

Using a DFT-based approach that treats the $5 f$ electrons relativistically, a Pu structure with zero net magnetic moment is arrived at by allowing the $5 \mathrm{f}$ orbital and $5 f$ spin moments to cancel each other. By combining the spin- and orbitalspecific Density of States (DOS) with state, spin and polarization specific transition moments, it is possible reconstruct the experimentally observed spectra from $\mathrm{Pu}$, obtained with linearly polarized soft x-rays. By extrapolating to a spin-resolving Fano configuration, it is shown how this would resolve the extant controversy over Pu electronic structure.

This work performed under the auspices of the U. S. Department of Energy by Lawrence Livermore National Laboratory under Contract DE-AC52-07NA27344.

PACS Numbers: 71.20Gj, 71.27.+a, 79.60.-I, 71.28+d., 72.25.Fe

*Corresponding Author: Tobin1@LLNL.Gov 
Despite substantial progress in the recent past, the exact nature of the electronic structure of Pu has not been resolved. While $\mathrm{Pu}$ is of immense technological and scientific importance and its phase diagram a study in both complexity and far-reaching impacts from seemingly trivial variations, there still is no consensus regarding its electronic structure. [Ref .1-5, and references therein] There have been several different avenues of attack upon the problem, but each seems to be beset with its own limitations and inherent flaws. For example, by postulating that the there is a long range magnetic ordering in $\mathrm{Pu}$, it is possible to explain the geometrical structure of all of the six different phases using the Density Functional Approach (DFT) within a modified Local Density Approximation. (LDA) [1] Unfortunately for that approach, the vast body of experimental data indicates that there is no long range magnetic ordering in $\mathrm{Pu}$. [2] To avoid the magnetism problem, some researchers suggested that Pu had a $5 f$ occupancy of nearly six, thus effectively filling the lower $5 f 5 / 2$ sub-shell and diminishing the likelihood of a magnetic moment. [3] Again unfortunately, this approach was also inconsistent with a substantial body of experimental evidence. In this case, X-ray Absorption Spectroscopy (XAS) and its cousin, Electron Energy Loss Spectroscopy (EELS), had been used to demonstrate the relativistic nature of the Pu $5 f$ states and that the total $5 f$ occupation was near to five. [4] Most recently, Shim, Haule and Kotliar argue that they can explain everything, using a DMFT approach that incorporates that best of the LDA/DFT and permits a non-magnetic solution that includes electron correlation. 
Here, an alternative picture for electron correlation in $\mathrm{Pu}$ is proposed. Using a Density Functional Theory based approach [6] that treats the $5 f$ electrons relativistically [4], a Pu structure with zero net magnetic moment is arrived at by allowing the $5 f$ orbital and $5 f$ spin moments to cancel out each other. To test this hypothesis, a direct comparison was made to extant Pu photoelectron spectra. However, rather than simply comparing the calculations themselves to the PES spectra, a process fraught with many pitfalls and inaccuracies, the spin and orbital specific calculations were used to generate simulated spectra, which were then compared to the experimental results. It is shown that by combining the spin- and orbital- specific Density of States (DOS) with state, spin and polarization specific transition moments [7], one can reconstruct the experimentally observed spectra from $\mathrm{Pu}$, obtained with linearly polarized soft $\mathrm{x}$ rays, [8] thus directly confirming the validity of this approach. (Figure 1) Furthermore, by extrapolating to a spin-resolving Fano experiment performed in a chiral configuration with a non-magnetic sample $[7,9,10]$, it is predicted what the spectral response of Pu should be and how this would be different that that expected for a Kondo shielded system. [9] The spectral modeling for the extrapolation was tested using the non-magnetic and highly relativistic system $\mathrm{Pt}$. (Figure 2) [10] It is proposed here that Fano measurements of Pu will permit the determination of the nature of electron correlation in Pu. (Figure 3)

The details of the computational method [6] and data collection [8] are described elsewhere. However, it should be noted that both the calculations and experiments face substantial hurdles. The calculations focus upon the $5 f$ 
electronic states in which itinerancy, hybridization, spin-orbit splitting and electron correlation interact complexly. $[1,6]$ The experiments are limited by the surface reactivity, complex phase diagram, toxicity and highly radioactive nature of the material. $[4,8]$

The calculations of the $m_{l}$ and $m_{s}$ specific density of states were made as follows. Including both spin-orbit splitting and orbital polarization [6], the variational principle was applied to a magnetically ordered $\delta$-Pu fcc lattice. Then, using this result as a starting point, the interatomic spacings were allowed to vary such that the $5 f$ orbital and $5 f$ spin moments were moved into perfect cancellation. Thus, each and every $\delta$-Pu in the fcc lattice was magnetically neutral, possessing neither long range magnetic ordering nor a permanent magnetic moment. The $m_{l}$ and $m_{s}$ specific density of states that were thusly generated are shown in the top panel of Figure 1.

Next, it is necessary to convert the $m_{l}$ and $m_{s}$ specific density of states into a simulated spectrum. To do this, the transition moments for each $m_{l}$ and $m_{s}$ state must be generated for the geometry specific to the Photoelectron Spectroscopy Experiment. In this case, the excitation was linearly polarized $x$ rays, incident at an angle of 60 degrees relative to the sample normal, with the polarization in the plane containing the incoming photons and the sample normal. The photoelectrons were collected along the sample normal. Using this geometry and a plane-wave-like final state, one can demonstrate that the state specific transition moments or intensities are those shown in the inset in the middle panel in Figure 1. [7] While the overall absolute intensity is photon energy 
dependent via the radial matrix elements, the relative intensities within the $5 f$ manifold are merely dependent upon the spherical harmonics involved. Thus, by truncating the $m_{\mathrm{I}}$ and $\mathrm{m}_{\mathrm{s}}$ specific density of states at the Fermi energy (only occupied states can emit) and multiplying by the transition moments shown in Figure 1 and summing, one arrives at the result shown in the middle panel of Figure 1, a simulated spectrum of $\mathrm{Pu}$. Comparing this to the experimental result shown in lowermost panel of Figure 1, it is clear that the simulated spectrum reconstructs the key features of the experimental data: the narrow maximum near the Fermi Energy, the minimum near $-1 / 2 \mathrm{eV}$ binding energy and the broad maximum near -1 to $-2 \mathrm{eV}$. (The results shown here in Figure 1 and in Figure 3 below are for a final state g-wave, which should dominate at these photon energies. Similar results can be obtained with a d-wave final state. [7])

Given the success of this method and the success of the previously reported DMFT approach [5], the question then becomes the following. Is there another level of information which will allow us to differentiate between these two hypothesized solutions? We believe there is and suggest that Fano measurements are the key to accessing this additional level of information.

Fano Spectroscopy is the measurement of spin resolved effects in nonmagnetic systems. First postulated in 1969 by Fano [11] and subsequently confirmed using an ion beam [12] and then photoelectron spectroscopy [13], It has recently been utilized to confirm the dynamically antiparallel alignment of quasiparticle (Kondo) and f- state (Lower Hubbard Band) electrons in Ce. [9] In Figure 2, the results for the Pt system are shown. [10] In the lowermost panel 
are the asymmetries $(A)$ for the two experimental configurations, one with the source to the left and the other with the source to the right.

$A=\{\operatorname{counts}($ up $)-\operatorname{counts}($ down $)\} /\{\operatorname{counts}($ up $)-\operatorname{counts}($ down $)\}$

The reversal of the spin dependency with the change in source confirms the Fano nature of these spin effects. From these asymmetries, one can generate a polarization (middle pane, Figure 2) and spin resolved spectra (top panel. Figure 2). The large spin-orbit splitting in the Pt $5 d$ states drives this spin dependence, which does not require a permanent magnetic moment nor long range magnetic ordering. If it is possible to see such an effect in the relativistic Pt $5 d$ states, why not in the relativistic Pu $5 f$ states?

The results of the simulation of the Pu Fano spectroscopy are shown in Figure 3. This is potentially the result for either a measurement with circularly polarized x-rays, where the measured spins are co-aligned with the helicity, or an experiment like that shown for Pt in Figure 2, where the spin measured is perpendicular to the plane containing the unpolarized Hel radiation and the chirality is derived from the orientation of the experimental vectors. $[9,10]$ Here, the dichroic intensities shown in the inset in Figure 3 are used along with the $m_{1}$ and $m_{s}$ specific density of states shown in Figure 1 . The product of this process is shown in the lower panel of Figure 3. Interestingly, there is very good agreement with the result predicted using an atomic model of $\mathrm{Pu}$ [7], shown in the upper panel of Figure 3. Moreover, these simulated spectra are very different than that observed for $\mathrm{Ce}$ [9], a known Kondo system. Thus, this experiment 


\section{should provide the detailed information necessary to distinguish between the two proposed models for electron correlation in $\mathrm{Pu}$.}

Finally, it is worthwhile to address the issue of the $4 d 5 / 2-4 d 3 / 2$ Branching Ratio (BR), which has been experimentally determined for Pu. [4] The experimental value is near 0.8 . The value derived from these calculated $m_{1}$ and $\mathrm{m}_{\mathrm{s}}$ specific density of states, using again state specific transition moments but this time for the XAS process, is 0.7 . Although close, the agreement is not exact. This is not surprising, since the process involves energy minimization in a magnetically ordered system, which has driven the system into a strong magnetically ordered limit. [7] The impact of this process can be seen in the inset in the top panel of Figure 3, where the DOS calculations for the non-spin orbit, spin-orbit, orbitally polarized and ferromagnetic cases are shown. Clearly, the ferromagnetic case effect has a strong perturbation upon the DOS. Despite this, the magnetic cancellation succeeds in reconstructing the valence PES spectra and achieves a fairly good match with the experimental BR result.

An alternative model for electron correlation in Pu has been proposed and it has been demonstated that it is consistent with the experimental PES results. A new experiment (Fano Spectroscopy) is proposed to resolve the Pu electronic structure controversy conclusively.

Lawrence Livermore National Laboratory is operated by Lawrence Livermore National Security, LLC, for the U.S. Department of Energy, National Nuclear Security Administration under Contract DE-AC52-07NA27344. Work that was performed by LLNL personnel was supported in part by the Office of Basic Energy Science at the U.S Department of Energy.- 
Figure Captions

Figure 1 Topmost panel: the $m_{\perp}$ and $m_{s}$ specific density of states from the magnetic cancellation calculations. Middle Panel: The simulated spectrum derived from the $m_{\perp}$ and $m_{s}$ specific density of states in the topmost panel and the state specific transition moments, shown in the inset. Lowermost panel: The experimental PES results for $\delta$-Pu, using linearly polarized x-rays as the excitation and with no spin detection.

Figure 2 The Fano Spectroscopy results for $\mathrm{Pu}$. Topmost panel: the spin resolved and spin integrated spectra, using unpolarized Hel photons as the excitation. The electrons were collected along the sample normal. The He I radiation was incident from either the left or the right side, at an angle of 45 degrees. The spin measured was perpendicular to the plane containing the $\mathrm{He}$ I radiation and the sample normal. Middle panel: the corresponding polarization. Lowermost panels: the asymmetries from the left and right Hel sources.

Figure 3 Simulated Fano Spectroscopy results for Pu. Lower panel: the result from the magnetic cancellation calculations, with the insets showing the dichroic state specific intensities. Top Panel: The simulation result from an atomic $5 f$ calculation. (See Ref 7.) The inset shows the DOS calculation results for non-magnetic Pu without a spin-orbit splitting (NM:No SO); nonmagnetic Pu with spin-orbit splitting in the $5 f$ states (NM: SO); non-magnetic Pu with both spinorbit splitting and orbital polarization (NM:SO + OP) and ferromagnetic Pu with both spin-orbit splitting and orbital polarization (FM:SO + OP) 


\section{References}

1. P. Söderlind and B. Sadigh, Phys. Rev. Lett. 92, 185702 (2004); B. Sadigh P. Söderlind and B. Wolfer Phys. Rev. B 68, 241101 (2003)P. Söderlind, A. Landa, and B. Sadigh, Phys. Rev. B 66, 205109 (2002).

2. J.C. Lashley, A. Lawson, R.J. McQueeney, and G.H. Lander, Phys. Rev. B 72, 054416 (2005).

3. L.V. Pourovskii, M.I. Katsnelson, A.I. Lichtenstein, L. Havela, T. Gouder, F. Wastin, A.B. Shick, V. Drchal, and G.H. Lander, EuroPhys. Lett. 74, 479 (2006); A.O. Shorikov, A.V. Lukoyanov, M.A. Korotin, and V.I. Anisimov, Phys. Rev. B 72, 024458 (2005); arXiv:condmat/0412724 v2 21 Jan 2005; A.B. Shick, V. Drchal, and L. Havela, EuroPhys. Lett. 69, 588 (2005).

4. J.G. Tobin et al, Phys. Rev. B 72, 085109 (2005); G. van der Laan et al, Phys. Rev. Lett. 93, 097401 (2004).K.T. Moore et al, Phys. Rev. Lett. 90, 196404 (2003).

5. J.H. Shim, K. Haule and G. Kotliar, Nature 446, 513 (2007).

6. P. Söderlind, "Quantifying the importance of orbital over spin correlations in $\delta$-Pu within density functional theory," Phys. Rev. B, in press, 2007.

7. J.G. Tobin, J. Alloys Cmpds 444-445, 154 (2007).

8. J.G. Tobin et al, Phys. Rev. B 68, 155109 (2003).

9. J.G. Tobin, S.W. Yu, T. Komesu, B.W. Chung, S.A. Morton, and G.D. Waddill, Europhysics Lett., 77, 17004 (2007).

10. S.-W. Yu and J.G. Tobin, Surface Science Letters, 2007, in press.

11. U. Fano, Phys. Rev. 178, 131 (1969); 184, 250 (1969).

12. J. Kessler and J. Lorenz, Phys. Rev. Lett. 24, 87 (1970); G. Baum, M.S. Lubell, and W. Raith, Phys. Rev. Lett. 25, 267 (1970).

13. U. Heinzmann, J. Kessler, and J. Lorenz, Phys. Rev. Lett. 25, 1325 (1970). 


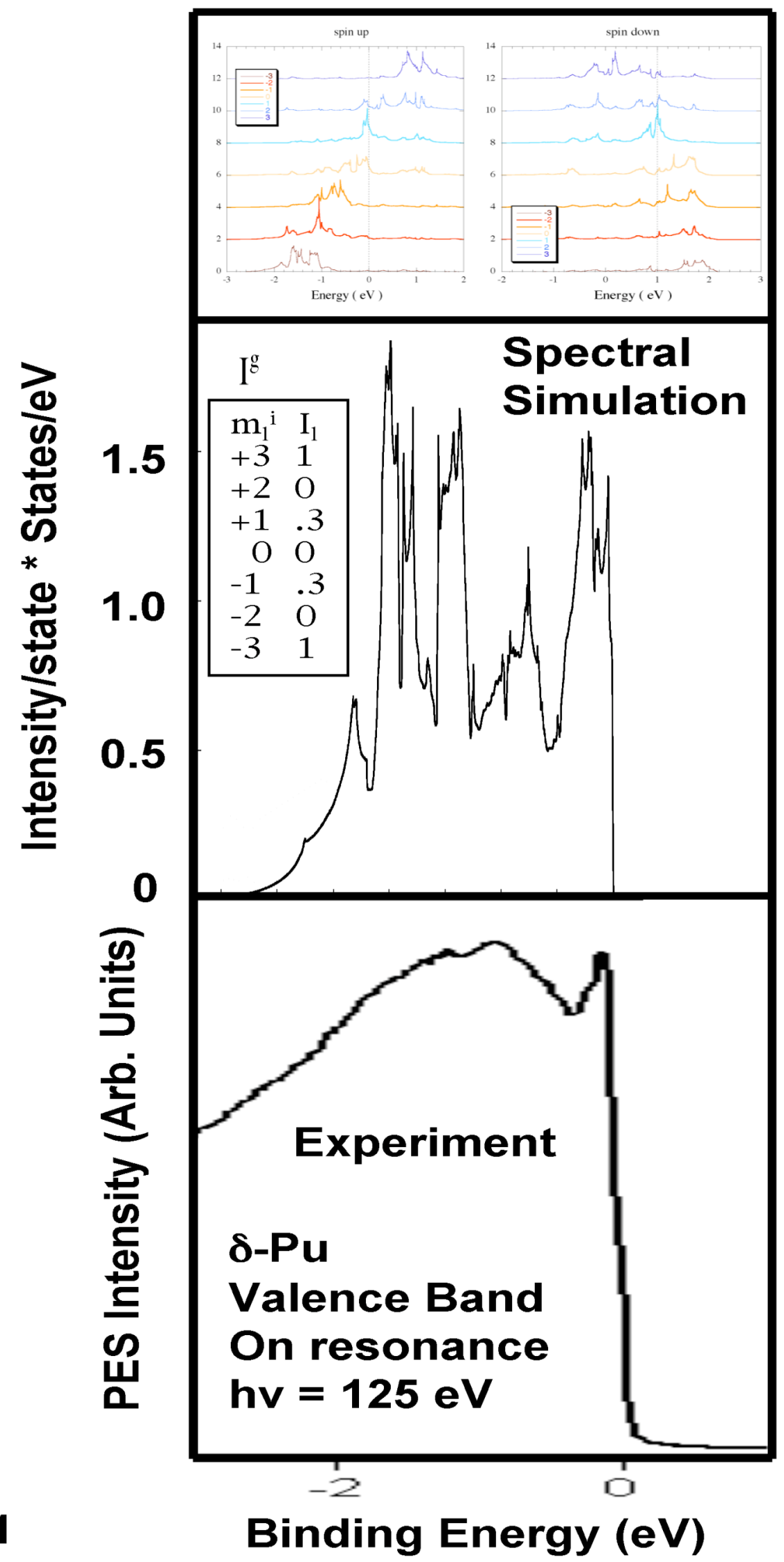

Figure 1 

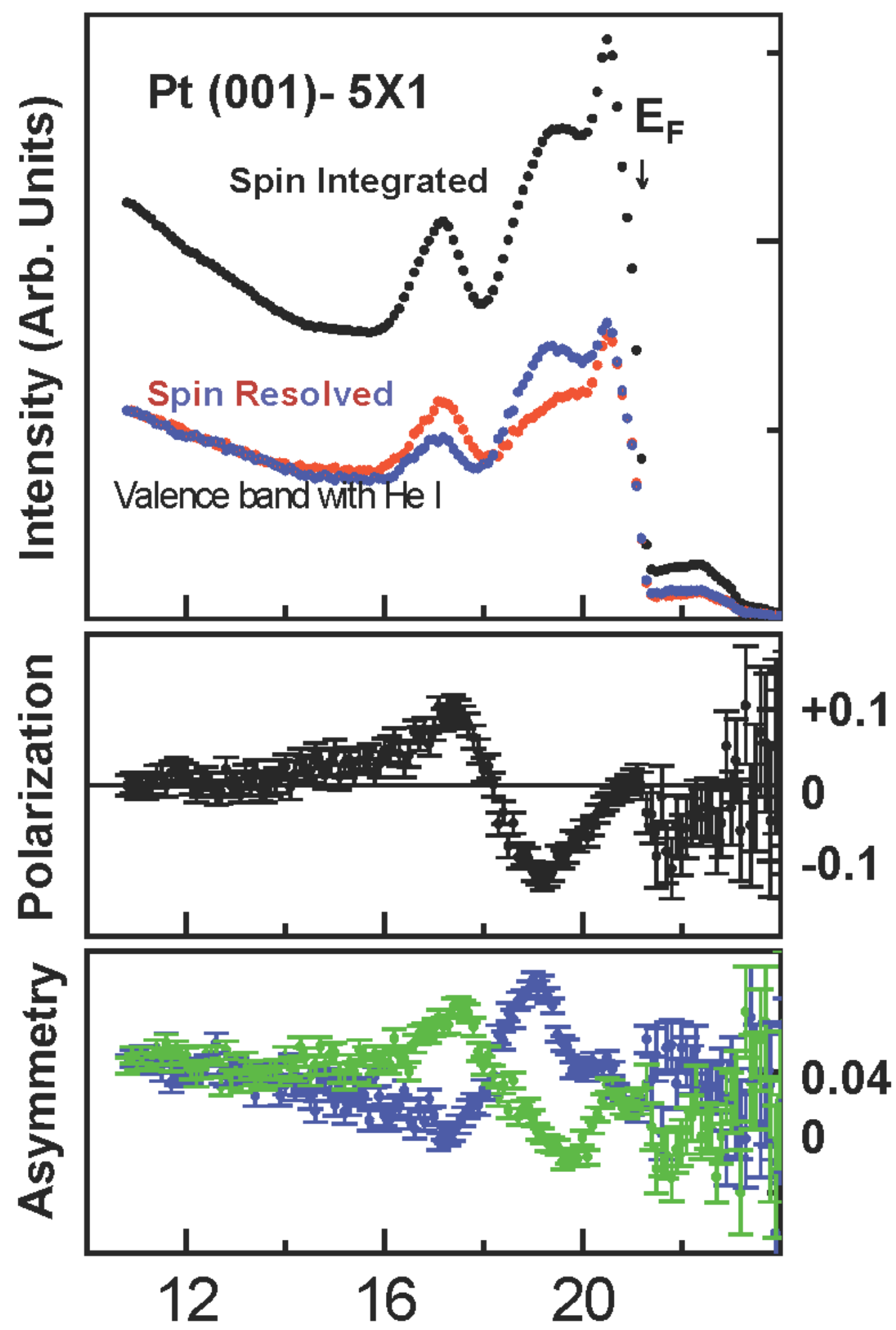

Figure 2 Kinetic Energy (eV) 

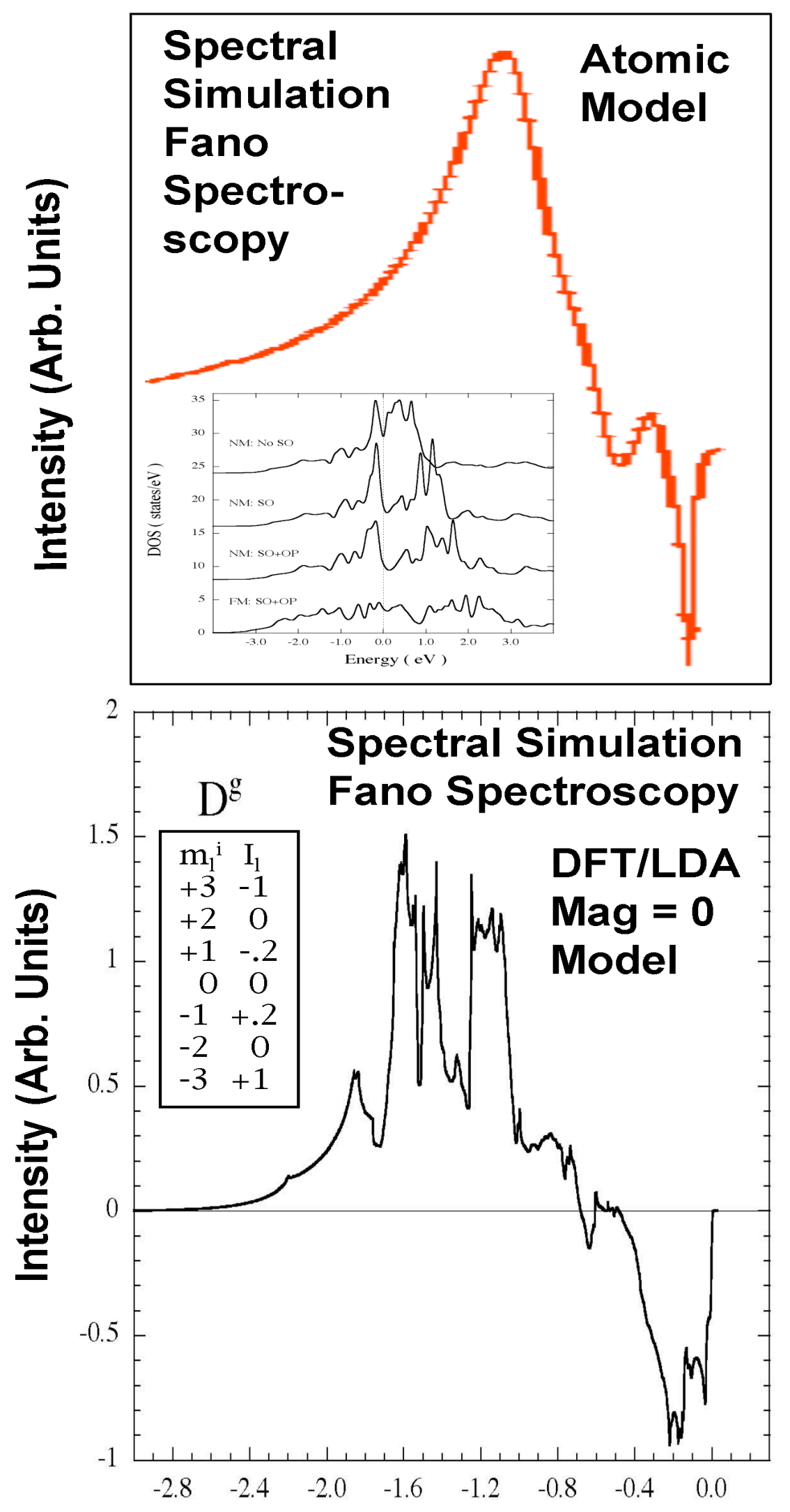

Figure 3

Binding Energy (eV) 